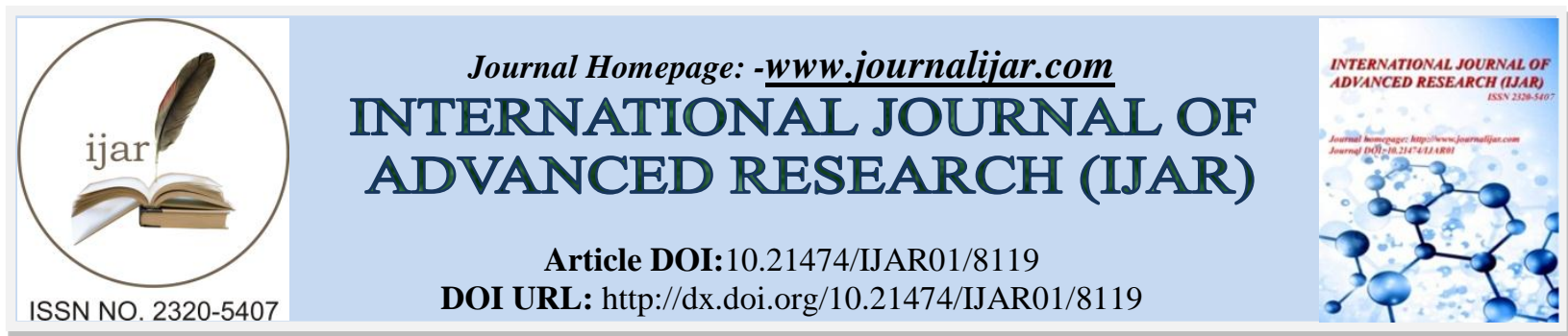

RESEARCH ARTICLE

\title{
LISTERIA MONOCYTOGENESIN RAW MILK AND DAIRY PRODUCTS; OCCURRENCE AND TRIALS FOR THEREDUCTION OF THEIR RESISTANCE TO ANTIBIOTICS.
}

\author{
Fatma I. El-hofy ${ }^{1}$, Ashraf A. Abd El-tawab ${ }^{2}$, Elham A. Mobarez ${ }^{3}$, Mohamed F. Abo El-Maati, Nancy Y. \\ Tawkol $^{4}$ and Ahmed A. Hefny ${ }^{4}$. \\ 1. Bacteriology, Immunology and Mycology Department, Faculty of Veterinary Medicine, Benha University, \\ 13736, Egypt. \\ 2. Pharmacology Department, Animal Health Research Institute, Dokki, Giza, 12618, Egypt. \\ 3. Biochemistry Department, Faculty of Agriculture, Zagazig University, 44511, Egypt. \\ 4. Veterinary Hospital, Faculty of Veterinary Medicine, Zagazig University, 44511, Egypt.
}

\section{Manuscript Info}

Manuscript History

Received: 01 October 2018

Final Accepted: 03 November 2018

Published: December 2018

Keywords:-

Listeria monocytogenes, raw milk, plant extracts, synergism.

\section{Abstract}

Listeria monocytogenes is a facultative intracellular pathogen responsible for severe infection in animals and humans. This study aimed to determine the occurrence of Listeria monocytogenes in 200 samples of raw milk and dairy products marketed in Egypt, with detection the antimicrobial resistance profiles of the isolates for different antimicrobial agents. The synergistic effects of three plant extracts in combination with the used antimicrobials to control resistant L. monocytogenes isolates were assessed, with evaluation of relationship between the antibacterial activity and composition of plant extracts. A total of $5(2.5 \%) \mathrm{L}$. monocytogenes isolates were identified ( 3 isolates from raw milk and two isolates from kariesh cheese and ice cream, one each). The isolates were sensitive to ciprofloxacin and sulphadimidine, while for amoxicillin and oxytetracycline, were resistant. The higher antimicrobial effects of basil and cinnamon extracts were detected, in addition, low concentrations of them were enough to show synergistic effect with the amoxicillin and oxytetracycline to control resistant isolates, these might be attributable to their chemical compositions. In conclusion, there is need for effectively pasteurized raw milk consumption or usage in the production of dairy products. In addition, the chemical composition of these plant extracts might help in its use to control resistant isolates with appropriate antibiotics without the need for increasing the used dosage doses.

Corresponding Author:-Ahmed A. Hefny. Address:- Veterinary Hospital, Faculty of Veterinary Medicine, Zagazig University, 44511, Egypt. 


\section{Introduction:-}

Listeriosis is a serious infectious disease of humans and animals, caused mainly by Listeria monocytogenes. The large outbreaks in humans were caused by consumption of raw orpost-pasteurization contaminated milk and mainly characterized by low incidence but high case fatality rate (Kasalica et al., 2011). L. monocytogenesis susceptible to most antibiotics, except for cephalosporins and fosfomycin. However, major concerns have been raised due to reports of Listeria spp. that are resistant to a wide range of antimicrobial agents that are commonly used for the treatment of listeriosis. Thus, as an alternative, combination therapy has been found more effectivebecause synergistic interactions can be faster in therapy, prevent emergence of resistance and increase efficacy of the used combinations (Marr et al., 2004). In addition, it can reduce toxicity, and provide broader spectrum of activity.

The presence of different antimicrobial compounds in plants help scientists to use these plants in treatment of some infectious diseases. However, combination therapy of various plant extracts with antibiotics exhibiting bactericidal synergism is a method leading tonew ways for treatment of infectious diseases (Chanda and Rakholiya, 2011).

The current study aimed to determine the prevalence of L. monocytogenes in raw milk and dairy products; soft cheese, kariesh cheese and ice cream at Qalyubiyah and Giza governorates, Egypt. The antimicrobial resistance profile of the isolates was determined against four different classes of antimicrobial agents. Moreover, the total phenolic compounds, total flavonoids compounds and antioxidant activity of three plant extracts were evaluated. Also, the synergistic effects of the three extracts in combination with the used antimicrobials to control resistant L. monocytogenes isolates were assessed.

\section{Materials and Methods:- \\ Bacterial isolates:-}

Isolation and identification

Two hundred random samples from fresh milk $(\mathrm{n}=80)$ and dairy products(soft cheese, kariesh cheeseand ice cream, 40 each) were obtained from different outlets at Qalyubiyah and Giza Governorates, Egypt, during the period of October 2016 to January 2017. The collected samples were aseptically transported in ice box as soon as possible to the laboratory for isolation and identification of L. monocytogenes according to ISO (2004).

For isolation of L. monocytogenes, 25 gramsof collected dairy products were pre-enriched in $225 \mathrm{ml}$ half strength Fraser broth (Oxoid - CM0895) for $24 \mathrm{~h}$ at $30^{\circ} \mathrm{C}$, then followed by second enrichment of $0.1 \mathrm{ml}$ of full strength Fraser broth for $48 \mathrm{~h}$ at $37^{\circ} \mathrm{C}$. Similarly, $25 \mathrm{ml}$ of collected fresh milk samples were passed the two step method for enrichment after $\mathrm{PH}$ adjusted to neutral. After the enrichment procedure, $0.1 \mathrm{ml}$ of the broth was streaked onto PALCAM agar (Oxoid CM0877) and incubated for $48 \mathrm{~h}$ at $37^{\circ} \mathrm{C}$. Listeria monocytogenes were preliminary identified by their colonial morphology on PALCAM agar. The suspected colonies were sub-cultured on Tryptone Soya Yeast Extract Agar (TSYEA; Sigma-Aldrish) and subjected to Gram's staining, microscopic examination, catalase test, and oxidase test. The isolates were also sub-culturedon semisolid medium (Nutrient broth supplemented with $0.5 \%$ agar, Oxoid) at $25^{\circ} \mathrm{C}$ for $12-18 \mathrm{~h}$ for the detection the typical tumbling motility (ISO, 2004). For further identification, Microgen ${ }^{\mathrm{TM}}$ Listeria-ID System (LAB; MID-67) was used according to the manufacturers' guidelines. The phenotypically isolates were confirmed using PCR targeting 16s rRNA(Kumar et al., 2015).

\section{Antimicrobial susceptibility testing:-}

Two-fold broth microdilution method was used to determine the minimum inhibitory concentrations (MIC) of theidentified strains against the antimicrobial agents amoxicillin, oxytetracycline, ciprofloxacin and sulphadimidine (Sigma-Aldrich), by cultivation in Mueller Hinton broth according to the Clinical and Laboratory Standards Institute guidelines (CLSI, 2012). There are no specific international interpretationcriteria for susceptibility testing of L. monocytogenesfor the examined agents. Therefore, the Staphylococcus spp. resistance breakpoints were used. The general resistance breakpoints of CLSI (2002) were used in case of amoxicillin, while, CLSI (2016) were used for tetracycline, ciprofloxacin and sulphadimidine. Resazurin dye as a redox indicator was used to detect the MIC values to give a high level of accuracy (Elshikh et al., 2016). Each isolate was examined in triplicate. The reference strain; L. monocytogenes (ATCC ${ }^{\circledR} 35152$ ) was used as a quality control. 


\section{Plant extracts:-}

Plant extracts preparation and analysis Three plant samples; cinnamon inner bark (Cinnamomum zylenticum), coriander leaves (Coriandrum sativum) and basil leaves (Ocimum basilicum), were obtained from Medicinal and Aromatic Plant Research Department, Horticulture Research Institute, Agricultural Research Center, Egypt. Preparation of the ultrasonicassisted ethanol 75\% extract from plants were applied according to El-Maati et al. (2016).

The plant extracts were analyzed for determination of total phenolic compounds (TPC) by a UV spectrophotometer using Folin-Ciocalteu reagent, and total flavonoid compounds (TFC) using the Folin-Ciocalteau method (Gülçin et al., 2010).

The antioxidant activity of the extracts was determined bymeasuring 1,1-diphenyl-2-picrylhydrazyl (DPPH') free radicalscavenging activity (Yamaguchi et al., 1998), $\beta$-Carotene-linoleic acid bleaching activity (Ordonez et al., 2006), and finally the ferric-reducing antioxidant power (FRAP) activity (Gülçin et al., 2010).

\section{MIC of ethanol plant extracts:-}

The influence of plant extracts was evaluated on clinical strainsbybroth microdilution method using the redox indicator resazurin as a visual marker of the MIC (Elshikh et al., 2016).

\section{Checkerboard assay:-}

Interactions between antibiotics and plant extracts were assessed by the broth dilution checkerboard MIC assay in Mueller Hinton broth. Different dilutions of each plant extract $(1 / 2,1 / 4$ and 1/8) were used separately with different concentrations of each antimicrobial agent (1/2,1/4 and 1/8 of the MIC value) according to Paparella et al. (2013). Where, the synergistic effect was assessed on the basis of the fractional inhibitory concentration (FIC) index representing the sum of the FICs ( $\Sigma$ FICs) of each drug tested, where the FIC derived from the lowest concentrations of extracts and the antibiotics in combination permitting no visible growth of the test organisms in the Mueller-Hinton broth after incubation for $24 \mathrm{~h}$ at 37 ${ }^{\circ} \mathrm{C}$. In addition, Kamatou et al. (2006) defined synergy to occur when $\Sigma$ FIC $<1.0$, additively when $\Sigma$ FIC $=1.0$ and antagonism when $\Sigma$ FIC $>1.0$.

$\Sigma$ FIC $=$ FIC $($ antibiotic $)+$ FIC (extract $)=($ MIC of extract in combination/MIC of extract alone $)+($ MIC of antibiotics in combination/MIC ofantibiotics alone)

\section{Results:-}

Bacterial isolates:-

Five isolates (2.5\%) from raw milk $(n=3)$, kariesh cheese $(n=1)$ and ice cream $(n=1)$ of L. monocytogenes were preliminary identified by their colonial morphology. On PALCAM agar, the colonies were small 2-3 mm gray-green surrounded by diffuse black zone (esculin hydrolysis), and on TSYEA agar; pinpoint colonies were observed. The isolates were further confirmed by microscopical and biochemical examination. Molecular confirmation of the five isolates by the amplification of $1200 \mathrm{bp}$ of the 16s rRNA gene; was also carried out.

The antimicrobial resistance profile determined by broth micro dilution method revealed that all L. monocytogenes isolates were resistant to amoxicillin and oxytetracycline, while, all the isolates were sensitive to ciprofloxacin and sulphadimidine.

\section{Plant extracts:-}

The best yield of extracts was obtained from (24.5\%) dry basil leave. The total phenolic compounds, total flavonoid compounds and antioxidant activity of all plant extracts were detected. The results revealed that TPC, TFC, and antioxidant activity was higher in basil leave and cinnamon inner bark extracts than coriander leave extract (Table 1). Regarding antimicrobial activity of plant extracts, the MICs against clinical isolates for dry basil leave, cinnamon inner bark and coriander leave extractsare reported in Table 2.

\section{Checkerboard assay:-}

The quantitative effects of different plant extracts with different concentrations in combination with different antibiotics with different concentrations are described as FIC index. Combinations of amoxicillin and oxytetracycline showed synergism with all plant extracts with different concentrations, while, nosynergism was detected in combinations with ciprofloxacin or sulphadimidine. The synergism of amoxicillin and oxytetracycline were detected only with (1/2 of the 
MIC value) high concentration of coriander, while, low concentrations of basil and cinnamon were needed to show the synergism. The most active concentrations between plant extracts and antibiotics with the fractional inhibitory concentration (FIC) index of each are reported in Table 2.

Table 1:-Extract Yield, TPC, TFC and Bio-Analytical Analysis Of Ethanol Plant Extracts

\begin{tabular}{|c|c|c|c|c|c|c|c|c|}
\hline \multirow[t]{2}{*}{ Plant extract } & \multirow{2}{*}{$\underset{(\%)}{\text { Extract yield }}$} & \multirow{2}{*}{$\underset{(\mathbf{m g ~ G A E} / \mathrm{g})}{\mathbf{T P C}}$} & \multirow{2}{*}{$\underset{(\mathrm{mg} Q \mathrm{QE} / \mathrm{g})}{\mathbf{T F C}}$} & \multirow{2}{*}{$\underset{(\%)}{\beta-C L A B}$} & \multirow[t]{2}{*}{ FRAP } & \multicolumn{3}{|c|}{$\underset{(\%)}{\text { DPPH` FRSA }^{\circ}}$} \\
\hline & & & & & & $0 \mathrm{~min}$ & $60 \mathrm{~min}$ & $120 \mathrm{~min}$ \\
\hline $\begin{array}{c}\text { Basil leave } \\
\text { (Ocimum basilicum })\end{array}$ & 24.5 & 186.49 & 77.2 & 79.2 & 1.49 & 80.4 & 79.0 & 79.1 \\
\hline $\begin{array}{c}\text { Cinnamon inner bark } \\
\text { (Cinnamomum zylenticum) }\end{array}$ & 10.42 & 116.49 & 75.2 & 74.2 & 1.042 & 74.7 & 78.6 & 76.5 \\
\hline $\begin{array}{c}\text { Coriander leaves } \\
\text { (Coriandrum sativum) }\end{array}$ & 8.675 & 20.24 & 10.0 & 12.4 & 0.179 & 13.7 & 26.7 & 31.1 \\
\hline
\end{tabular}

TPC; total phenolic compounds, TFC; total flavonoid compounds, $\boldsymbol{\beta}$-CLAB; $\beta$-Carotene/linoleic acid bleaching which measured after $2 \mathrm{~h}$ and compared with TBHQ; tertiary butylhydroquinone (as positive control), FRAP; Ferric reducing antioxidant power compared with TBHQ (as positive control), DPPH` FRSA; 1,1-diphenyl-2-picrylhydrazyl free radicalscavenging activity which measured at 0 -time, 60 and 120 minutes

Table 2:-MIC Of Used Antibiotics And Plant Extracts, With The Most Active Combination

\begin{tabular}{|c|c|c|c|c|c|c|c|c|c|c|c|c|c|c|c|c|c|c|c|}
\hline \multirow{3}{*}{ Isolate } & \multicolumn{4}{|c|}{$\begin{array}{c}\begin{array}{c}\text { MIC of antimicrobials } \\
(\mu \mathrm{g} / \mathrm{ml})\end{array} \\
\end{array}$} & \multicolumn{3}{|c|}{$\begin{array}{c}\text { MIC of plant extracts } \\
(\mathrm{mg} / \mathrm{ml})\end{array}$} & \multicolumn{6}{|c|}{$\operatorname{Amx}$} & \multicolumn{6}{|c|}{ Oxy } \\
\hline & \multirow{2}{*}{$A \mathbf{m x}$} & \multirow{2}{*}{ Oxy } & \multirow{2}{*}{$\mathrm{Cip}^{1}$} & \multirow{2}{*}{ Sul $^{1}$} & \multirow{2}{*}{ Cin } & \multirow{2}{*}{ Cor } & \multirow{2}{*}{ Bas } & \multicolumn{2}{|c|}{ Cin } & \multicolumn{2}{|c|}{ Cor } & \multicolumn{2}{|c|}{ Bas } & \multicolumn{2}{|c|}{ Cin } & \multicolumn{2}{|c|}{ Cor } & \multicolumn{2}{|c|}{ Bas } \\
\hline & & & & & & & & $\mathrm{MIC}^{2}$ & FICI & MIC & FICI & MIC & FICI & MIC & FICI & MIC & FICI & MIC & FICI \\
\hline Strain & 32 & 32 & 1 & 128 & 12.5 & 200 & 12.4 & $8^{\mathrm{A}}+1$ & 1). 250 & $8^{\mathrm{A}}+1$ & .625 & $8^{\mathrm{A}}+1$ & 1.375 & $8^{\mathrm{A}}+1$ & 1.375 & $\left(8^{\mathrm{A}}+1\right.$ & 1.625 & $\left(8^{\mathrm{A}}+\right.$ & 1.250 \\
\hline Strain & 16 & 32 & 0.5 & 64 & 25 & 200 & 12.5 & $8^{\mathrm{A}}+1$ & 11.625 & $4^{\mathrm{A}}+1$ & .750 & $A^{\mathrm{A}}+1$ & 1.750 & $8^{\mathrm{A}}+1$ & .250 & $4^{\mathrm{A}}+1$ & 1.750 & $\left(8^{\mathrm{A}}+1\right.$ & 1.250 \\
\hline Strain & 32 & 16 & 1 & 64 & 12.5 & 200 & 12.5 & $8^{\mathrm{A}}+1$ & & $88^{\mathrm{A}}+1$ & .625 & & .375 & $8^{\mathrm{A}}+1$ & .625 & $\left(4^{\mathrm{A}}+\right.$ & $\begin{array}{r}0.75 \\
(\mathrm{~S})\end{array}$ & & .750 \\
\hline Strain & 128 & 64 & 1 & 128 & 25 & 200 & $6.2=$ & $8^{\mathrm{A}}+1$ & 11.375 & $88^{\mathrm{A}}+1$ & .625 & $8^{\mathrm{A}}+1$ & .375 & $8^{\mathrm{A}}+1$ & .375 & $\left(8^{\mathrm{A}}+1\right.$ & 1.625 & $\left(8^{\mathrm{A}}+1\right.$ & 1.375 \\
\hline Strain & 64 & 32 & 1 & 128 & 25 & 100 & 12.5 & & & & .7 & & 1.500 & & .250 & $\left(4^{\mathrm{A}}+1\right.$ & 1.750 & $\left(8^{\mathrm{A}}+\right.$ & 1.375 \\
\hline
\end{tabular}

No synergism was reported between all examined plant extracts with different concentration of ciprofloxacin and sulphadimidine, ${ }^{2}$ The most active combination $\left(1 / 8^{\mathrm{A}}+1 / 4^{\mathrm{P}}\right)$ means $1 / 8$ concentration of MIC of antibacterial agent $+1 / 4$ concentration of MIC of plant extract, ${ }^{3} \mathrm{FICI}$; the fractional inhibitory concentration index, Amx; amoxicillin, Oxy; oxytetracycline, Cip; ciprofloxacin, Sul; sulpadimidine, Cin; cinnamon, Cor; coriander, Bas; basil, Remarks; (S) means Synergism

\section{Discussion:-}

L.monocytogenes is one of the important emerging bacterial zoonotic diseases worldwide. It is associated with consumption of raw milk or dairy products made from unpasteurized milk (Dewé, 2014). The current studyaimed to investigate the occurrence of L. monocytogenes in raw milk, and dairy products (soft cheese, kariesh cheese and ice cream), obtained from retail outlets at Qalyubiyah and Giza Governorates, Egypt, and to determine the antimicrobial resistance profile. Furthermore, the current study evaluated a trial to reduce the resistance of multidrug resistantisolates by using different plant extracts which have antimicrobial effect with different concentrations.

The isolation rate of L. monocytogenesin raw milk samples was 3.75\%. In accordance, Meshref et al. (2015) reported that the prevalence rates of L. monocytogenes in raw milk samples was 3.92\%. However, fresh cow milk samples were free from L. monocytogenes in another study (Al Kassaa et al., 2016). Infected animals, poor silage quality and insufficient 
hygienic conditions during milking and storage are likely the most common causes of L. monocytogenes contamination in raw milk.

The Kariesh cheese is a home-made cheese made in Egypt and Palestine. The current study showed that the prevalence of L. monocytogenes was $2.5 \%$ in Kariesh cheese samples. Variable isolation rates of L. monocytogenes in Kariesh cheese were reported in Egypt; 20\% (Hussien et al., 2013) and 6\% (Abd El Tawab et al., 2015). On the other hand, L. monocytogenes was not detected in soft cheese samples. The same results were recorded in Egypt (Ahmed, 2013), while in Costa Rica, Chaves and Arias (2009) reported the isolation of 27 L. monocytogenes isolates from 110 soft cheese samples. Regarding, ice cream samples; the prevalence rate of L. monocytogenes was $2.5 \%$. Similar result was reported in Egypt (Tantawy, 2011). On contrary, high prevalence rate (26\%) was reported in Greece (Effimia, 2015). The prevalence of L. monocytogenes in dairy products might reveal insufficient heat treatment of contaminated milk and improper sanitation of equipment.

The increased rate of L. monocytogenes resistance to one or more clinically relevant antibiotics has been reported (Morvan et al., 2010). The current study revealed that all isolates were sensitive to both ciprofloxacin and sulphadimidine. Similar results were previously reported (Karadal and Yildirim, 2014). However, all L. monocytogenes in the current study were resistant to amoxicillin and oxytetracycline. In accordance, Peter et al. (2016) reported that 16 L. monocytogenes isolates in Nigeria were resistant to amoxicillin and tetracycline. High resistance rates to amoxicillin and oxytetracycline may be partly attributable to overuse of these antibiotics in veterinary field, which increase genesmutation rate, in addition to acquisition of mobile genetic elements.

The continuous increase in microbial resistance to different types of antibiotics increases the need to find new classes of antibiotics. Antimicrobials of plant origin may give a hope to find a solution to this problem. Phenolic compounds are mainly considered as scavengers of free radicals (Gülçin et al., 2005). The bio-analytical analysis of plant extracts used in the current study demonstrated that basil and cinnamon had higher amount of TPC than coriander. In accordance, the high amount of TPC inbasil and cinnamon were reported (Snigdha and Monika, 2014). Also, higher amounts of TFC were reported in the current study than coriander. Similar finding was reported (Jangra et al., 2018).

The ability of natural antioxidants to inhibit the growth of bacterial pathogens has been documented to have influence on infectious diseases (Roginsky and Lissi, 2005). In the current study, the highest antioxidant activity was detected in basil followed by cinnamon then coriander. The antimicrobial activities of the ethanol extract of plant extracts against bacterial isolates were determined by evaluating the presence of MIC values, and thus, their potencies were assessed quantitatively (Yildiz, 2016). In the current study, the higher antimicrobial effects of basil and cinnamon were detected. These results might be conducted to the positive relationship between antibacterial activity and composition of plant extracts from TPC and TFC, in addition to antioxidant activity of plant extracts (Yildiz, 2016). The yield of extracts recovered from the examined dried plants in the current study ranged from 8.675 to $24.5 \%$. The variation in the extraction yields from different plants is attributed to their varied chemical composition, efficiency of extraction and the surrounding climatic conditions, in addition to the phytochemical properties and differences among bacterial species (Brant et al., 2008). Also, the variations in the ability of antibiotics and plant extracts combinations to reduce drug resistant in bacteria might be conducted to their effect on efflux pumps, which need to be evaluated (Wagner and Ulrich-Merzenich, 2009).

The interaction between antibiotics and bioactive plant extracts is a promising concept (Adwan and Mhanna, 2008). The development of improved management of microbial infections depends mainly on the clear differentiation of synergistic from antagonistic interactions. It was assessed on the basis of the fractional inhibitory concentration (FIC) index. The antimicrobial activities of basil, cinnamon and coriander extracts alone were evaluated (Shaaban et al., 2013). Betoni et al. (2006) reported that although the antimicrobial activity of a plant extract has not been relatively high, the synergism rates with other antibiotics were relatively high. In the current study, low concentrations of amoxicillin and oxytetracycline showed synergism with all plant extracts used against resistant isolates, especially, with basil or cinnamon extracts showed relatively high synergism than coriander extract. These findings confirmed that the activity of antibacterial combinations were more effective than individual agents. The same findings were reported in other studies. These results are consistent with a previous study mentioned that the double attack of two agents on different target sites of the bacteria could theoretically lead to synergistic effect (Olajuyigbe and Afolayan, 2012). For instance, agents that inhibitprotein synthesis (tetracycline) or interfere with cell wall synthesis (amoxicillin) had strongest synergistic effect with plant extracts (Betoni et al., 2006; Marzoog, 2013). Also, the antagonistic effect in vitro between coriander in combination with amoxicillin 
/clavulanic acid against L. monocytogeneswas reported (Shaaban et al., 2013). Ciprofloxacin and sulphadimidine showed neither synergistic nor additive effect against L. monocytogenes in the current study with the investigated plants. These results are consistent with those obtained byBetoni et al. (2006) who reported that none or weak synergism activity between nucleic acid synthesis inhibitors and plant extracts. In contrary, Shaaban et al. (2013) reported antagonistic effect between norfloxacin (Quinolones) and coriander. The absence of effect between plant extracts and ciprofloxacin or sulphadimidine in the current study might be because the isolates were sensitive to them. In conclusion, this study indicated that raw milk and dairy productscould be considered as sources of L. monocytogenes. Therefore, raw milk must be effectively pasteurized before consumption or usage in the production of dairy products. The chemical characters of ethanol extracts of basil, cinnamon and coriander, including; TPC, TFC and anti-oxidant properties, was in parallel to their synergistic effect with antibiotics. Furthermore, their concomitant administration together could be useful in treating L. monocytogenes infectionswith minimal concentrations of antibiotics, and subsequently minimal side effects from antibiotics.

\section{Disclosure Statement:-}

No competing financial interests exist.

\section{References:-}

1. Abd El Tawab, A., A. Maarouf and Z. Mahdy (2015): Bacteriological and molecular studies of Listeria species in milk and milk products at El-Kaliobia governorate. BVMJ, 2(29): 170-181.

2. Adwan, G. and M. Mhanna (2008): Synergistic effects of plant extracts and antibiotics on Staphylococcus aureus strains isolated from clinical specimens. Middle-East Journal of Scientific Research, 3(3): 134-139.

3. Ahmed, H. (2013): Public health significance of Listeria as a foodborne pathogen. Zoonoses Department. Faculty of veterinary medicine Cairo University, Egypt.

4. Al Kassaa, I., K. El Omari, B. Esmail, M. Hamze and M. Saati (2016): Prevalence of Listeria monocytogenes in raw milk in North Lebanon. LSJ, 37-43.

5. Betoni, J.E.C., R.P. Mantovani, L.N. Barbosa, L.C. Di Stasi and A. Fernandes Junior (2006): Synergism between plant extract and antimicrobial drugs used on Staphylococcus aureus diseases. Memórias do Instituto Oswaldo Cruz, 101(4): 387-390.

6. Brant, R., J. Pinto, S. Bertolucci and C. Albuquerque (2008): Essential oil content of Aloysia triphylla (L'Her.) Britton in function of seasonal variation. Rev. Bras. Pl. Med, 1083-88.

7. Chanda, S. and K. Rakholiya (2011): Combination therapy: Synergism between natural plant extracts and antibiotics against infectious diseases. Microbiol Book Series, 520-529.

8. Chaves, C. and M.L. Arias (2009): Characterization of Listeria monocytogenes isolates obtained from raw cheese samples acquired from different Costa Rican producer zones. Archivos latinoamericanos de nutricion, 59(1): 66-70.

9. 9. CLSI (2002): Performance standards for antimicrobial susceptibility testing, vol. 22, no. 1. 12th informational supplement M100-S12. Wayne, PA. Clinical and Laboratory Standards Institute.

10. CLSI (2012): Methods for Dilution Antimicrobial Susceptibility Tests for Bacteria That Grow Aerobically; Approved Standard-Ninth Edition. CLSI document M07-A9. Wayne, PA. Clinical and Laboratory Standards Institute.

11. CLSI (2016): Performance Standards for Antimicrobial Susceptibility Testing. 26th ed. CLSI supplement M100S. Wayne, PA. Clinical and Laboratory Standards Institute.

12. Dewé, T. (2014): Report on the rapid integrated assessment of nutrition and health risks in small ruminant value chains in Ethiopia. Nairobi, Kenya: ILRI.

13. Effimia, E. (2015): Prevalence of Listeria monocytogenes and Salmonella spp. Ready-to-Eat Foods in Kefalonia, Greece. J Bacteriol Parasitol, 6(243): 2.

14. El-Maati, M.F.A., S.M. Labib, A.M. Al-Gaby and M.F. Ramadan (2016): Antioxidant and antibacterial properties of different extracts of garden cress (Lepidium sativum L.). Zagazig J. Agric. Biochem. Appl., 43(5): 1685-1697.

15. Elshikh, M., S. Ahmed, S. Funston, P. Dunlop, M. McGaw, R. Marchant and I.M. Banat (2016): Resazurin-based 96well plate microdilution method for the determination of minimum inhibitory concentration of biosurfactants. BIOTECHNOL LETT, 38(6): 1015-1019.

16. Gülçin, İ., Ş. Beydemir, G. Şat and Ö.İ. Küfrevioğlu (2005): Evaluation of antioxidant activity of cornelian cherry (Cornus mas L.). Acta alimentaria, 34(2): 193-202. 
17. Gülçin, I., E. Bursal, M.H. Şehitoğlu, M. Bilsel and A.C. Gören (2010): Polyphenol contents and antioxidant activity of lyophilized aqueous extract of propolis from Erzurum, Turkey. Food Chem Toxicol, 48(8-9): 2227-2238.

18. Hussien, M.F., M.M. Amin and O.A. Sadek (2013): Comparison between the microbiological quality of kariesh cheese manufactured from raw and pasteurized skimmed milk sold in Assiut city markets. Assiut Vet. Med. J, 59(138): 129-137.

19. ISO (2004): Microbiology of food and animal feeding stuffs - Horizontal method for the detection and enumeration of Listeria monocytogenes - Part 1: Detection method. International Standard Organization ISO 11290-1, AMENDMENT 1: Modification of the isolation media and the haemolysis test, and inclusion of precision data. Geneva, Switzerland.

20. Jangra, S.S., V.K. Madan and I.D. Singh (2018): Comparative analysis of phytochemical profile and antioxidant activity of coriander (Coriandrum sativum L). Asian J. Chem, 30508-512.

21. Kamatou, G., A. Viljoen, S. Van Vuuren and R. Van Zyl (2006): In vitro evidence of antimicrobial synergy between Salvia chamelaeagnea and Leonotis leonurus. S Afr J Bot, 72(4): 634-636.

22. Karadal, F. and Y. Yildirim (2014): Antimicrobial susceptibility and serotype distribution of Listeria monocytogenes isolates obtained from raw milk cheese samples sold in Nigde. studies, 2627.

23. Kasalica, A., V. Vuković, A. Vranješ and N. Memiši (2011): Listeria monocytogenes in milk and dairy products. Biotech Anim Husbandry, 27(3): 1067-1082.

24. Kumar, A., S. Grover and V.K. Batish (2015): Exploring specific primers targeted against different genes for a multiplex PCR for detection of Listeria monocytogenes. 3 Biotech, 5(3): 261-269.

25. Marr, K.A., M. Boeckh, R.A. Carter, H.W. Kim and L. Corey (2004): Combination antifungal therapy for invasive aspergillosis. Clin Infect Dis, 39(6): 797-802.

26. Marzoog, T.R. (2013): Synergistic Effect of Rosmarinus officinalis Extract with Antibiotics against Different Bacterial Isolates. JET, 31(5 Part (B) Scientific): 678-686.

27. Meshref, A., M. Zeinhom and N.S. Abdel-Atty (2015): Occurrence and Distribution of Listeria Species in Some Egyptian Foods. AJVS, 46(1).

28. Morvan, A., C. Moubareck, A. Leclercq, M. Hervé-Bazin, S. Bremont, M. Lecuit, P. Courvalin and A. Le Monnier (2010): Antimicrobial resistance of Listeria monocytogenes strains isolated from humans in France. Antimicrob Agents Chemother, 54(6): 2728-2731.

29. Olajuyigbe, O.O. and A.J. Afolayan (2012): Synergistic interactions of methanolic extract of Acacia mearnsii De Wild. with antibiotics against bacteria of clinical relevance. IJMS, 13(7): 8915-8932.

30. Ordonez, A., J. Gomez and M. Vattuone (2006): Antioxidant activities of Sechium edule (Jacq.) Swartz extracts. Food Chem, 97(3): 452-458.

31. Paparella, A., A. Serio, C. Chaves-López and G. Mazzarrino (2013): Plant-based intervention strategies for Listeria monocytogenes control in foods. Microbial pathogens and strategies for combating them: Science, technology and education, 21230-1246.

32. Peter, A., E. Umeh, E. Azua and G.A. Obande (2016): Prevalence and Antimicrobial Susceptibility of Listeria monocytogenes Isolated from Beef, Pork and Chicken Sold in Makurdi Metropolis. BMRJ, 14(5): 1-7.

33. Roginsky, V. and E.A. Lissi (2005): Review of methods to determine chain-breaking antioxidant activity in food. Food Chem, 92(2): 235-254.

34. Shaaban, H., M. Ahmed, L. Sideek and M. Amer (2013): Study on the antimicrobial activity and Synergistic/antagonistic effect of interactions between antibiotics and some spice essential oils against pathogenic and food-spoiler microorganisms. JASR, 9(8): 5076-5085.

35. Snigdha, C. and T. Monika (2014): Effect of thermal processing on total phenolic content and antioxidant activity of Coriandrum sativum L. leaves. AJBS, 9(1): 58-62.

36. Tantawy, H. (2011): Listeria monocytogenes in Egyptian milk and dairy products. Milk Hygiene Department. Faculty of veterinary medicine Alexandria University, Egypt.

37. Wagner, H. and G. Ulrich-Merzenich (2009): Synergy research: approaching a new generation of phytopharmaceuticals. Phytomedicine, 16(2-3): 97-110.

38. Yamaguchi, T., H. Takamura, T. Matoba and J. Terao (1998): HPLC method for evaluation of the free radicalscavenging activity of foods by using 1, 1-diphenyl-2-picrylhydrazyl. Biosci Biotechnol Biochem, 62(6): 1201-1204.

39. Yildiz, H. (2016): Chemical composition, antimicrobial, and antioxidant activities of essential oil and ethanol extract of Coriandrum sativum L. leaves from Turkey. INT J FOOD PROP, 19(7): 1593-1603. 